\title{
A calculation of radiative lifetimes of electronically excited rovibronic levels of $\mathrm{CuF}$
}

\author{
J. Schamps, J.M. Delaval and O. Faucher \\ Laboratoire de Spectroscopie Moléculaire, Unité de Recherche Associée au CNRS No. 779, "Molécules Diatomiques", \\ Université des Sciences et Techniques de Lille Flandres Artois, UFR de Physique, Bâtiment P5, 59655 Villeneuve d'Ascq Cedex, \\ France
}

\begin{abstract}
Radiative lifetimes for rovibronic levels of the excited valence states of $\mathrm{CuF}$ are calculated using a model including both spinorbit and Coriolis interactions. According to a discussion concerning their decay mechanisms, the spin-orbit components are classified into three classes with lifetimes differing by several orders of magnitude. Electronic transition intensity patterns are calculated and compared with spectroscopic observations.
\end{abstract}

\section{Introduction}

In a previous paper [1] electronic transition dipole moments coupling the spin-orbit components of the valence states of $\mathrm{CuF}$ of $\mathrm{Cu}^{+}\left(3 d^{9} 4 s\right) \mathrm{F}^{-}\left(2 p^{6}\right)$ structure with the $\mathrm{X}^{1} \boldsymbol{\Sigma}^{+}$ground state and - typically one order of magnitude smaller - electronic transition moments inter-coupling these excited spin-orbit components have been calculated and tabulated ( $\mathrm{ta}$ ble 6 of ref. [1]). Radiative lifetimes of the valence excited states have been deduced from these transition moments. However this work was restricted to the spin-orbit coupling approximation, i.e. only spinorbit interaction was taken into account in perturbing the Born-Oppenheimer electronic states. Under these conditions, it was shown that radiative decay of all the $\Omega=1(\mathrm{e}, \mathrm{f})$ components essentially took place towards the $\mathrm{X}^{1} \Sigma^{+}$ground state while the other components $(\Omega=0(f), 2(e, f)$ and $3(e, f))$ decayed much more slowly towards lower-lying excited valence states. But these last components could also radiate towards the ground state if Coriolis coupling, that mixes rovibronic levels of different $\Omega$-components through rotation-electronic and spin-rotation interactions, is added to the model. Indeed these radiative channels are expected to play a significant part in the lifetimes of several of these states and Coriolis coupling need be taken into account. New wavefunc- tions were therefore determined by diagonalizing the total Hamiltonian matrix including the Coriolis part of the rotation Hamiltonian. Their reliability was successfully tested by calculating $\Lambda$-splitting magnitudes in the excited states [2]. Fhe very good agreement with spectroscopic data prompted us to use these wavefunctions in further refined calculations of CuF excited state lifetimes.

\section{Lifetime calculations}

In comparison with the spin-orbit case treated in ref. [1], in which only one transition moment is involved for each state, the mixing of several basis functions with different $\Omega$ values in the new spin-orbit-Coriolis wavefunctions makes it necessary to modify formula (2) of ref. [1]. In view of this, the perturbed wavefunctions are developed in terms of the unperturbed Born-Oppenheimer basis functions:

$$
\begin{aligned}
& |\tilde{n}(\tilde{S} \tilde{\Lambda} \tilde{\Omega}) ; v ; \tilde{\Omega} J \boldsymbol{l}\rangle\rangle \\
& \quad=\sum_{n} C_{\tilde{n} n}|n(S \Lambda \Omega) ; v, \Omega J M \epsilon\rangle .
\end{aligned}
$$

Here, the tildes over the $S, \Lambda, \Omega$ symbols [3] express the fact that, after matrix diagonalization, these symbols do not designate rigorously good quantum numbers but only "predominant" ones; $n$ (and $\tilde{n}$ ) is an 
integer number (or even a letter in spectroscopy) to distinguish between different states having same $S$, $\Lambda, \Omega$ numbers; $\epsilon$ is an index to distinguish between $\mathrm{e}$ $(\epsilon=+1)$ and $f(\epsilon=-1)$ levels. As explained in ref. [2], the $C_{\tilde{n} n}$ coefficients are obtained from diagonalization of the energy matrix so as to best fit the location of the as yet spectroscopically observed states.

Within the electronic dipole approximation (the only one used in this paper except otherwise stated) the transition probability, $A$ (in $\mathrm{s}^{-1}$ ), for a spontaneous radiative transition from an excited state $\left(\tilde{n}^{\prime}\right.$ $\left.\tilde{\Omega^{\prime}} v^{\prime} J^{\prime} \epsilon^{\prime}\right)$ of energy $E^{\prime}$ towards a lower-lying state ( $\tilde{n}^{\prime \prime}$ $\widetilde{\Omega}^{\prime \prime} v^{\prime \prime} J^{\prime \prime} \epsilon^{\prime \prime}$ ) of energy $E^{\prime \prime}$ is written [4-7]:

$$
\begin{aligned}
& A_{\tilde{n}^{\prime} \Omega^{\prime} v^{\prime} J^{\prime} \epsilon^{\prime} ; \tilde{n}^{\prime \prime} \Omega^{\prime \prime} v^{\prime \prime} J^{\prime \prime} \epsilon^{\prime \prime}}=\frac{16 \pi^{3}}{3 h \epsilon_{0} c^{3}} \nu_{\tilde{n}^{\prime} \boldsymbol{\Omega}^{\prime} v^{\prime} J^{\prime} \epsilon^{\prime} ; \tilde{n}^{\prime \prime} \bar{\Omega}^{\prime \prime} v^{\prime \prime} J^{\prime \prime} \epsilon^{\prime \prime}}^{3} \\
& \times \frac{S_{n^{\prime} \Omega^{\prime} v^{\prime} J^{\prime} \epsilon^{\prime} ; \hbar^{\prime \prime} \hat{\Omega}^{\prime \prime} \nu^{\prime \prime} J^{\prime \prime} \epsilon^{\prime \prime}}}{2 J^{\prime}+1}
\end{aligned}
$$

where $\nu$ is the frequency and $S$ is the line strength of the rovibronic transition [3], i.e. the sum of the squared modulae of the dipole moment matrix elements over all the $M^{\prime}$ and $M^{\prime \prime}$ sublevels of the upper and lower states expressed in the Kronig-symmetrized basis set (table 2 of ref. [2]). Some manipulation leads to:

$$
\begin{aligned}
& S_{\tilde{n}^{\prime} \Omega^{\prime} V^{\prime} J^{\prime} \varepsilon^{\prime} ; n^{\prime \prime} \Omega^{\prime \prime} \nu^{\prime \prime} J^{\prime \prime} \varepsilon^{\prime \prime}} \\
& \quad=\left.\left(2 J^{\prime \prime}+1\right)\right|_{n^{\prime} \Omega^{\prime}, n^{\prime \prime} \Omega^{\prime \prime}} \sum_{q} C_{\tilde{n}^{\prime} n^{\prime}} C_{\tilde{n}^{\prime \prime} n^{\prime \prime}} \\
& \quad \times\left.\left(\begin{array}{crc}
J^{\prime} & 1 & J^{\prime \prime} \\
\Omega^{\prime} & -q & -\Omega^{\prime \prime}
\end{array}\right)\left\langle n^{\prime} \Omega^{\prime} v^{\prime}\left|\mu^{q}\right| n^{\prime \prime} \Omega^{\prime \prime} v^{\prime \prime}\right\rangle\right|^{2} .
\end{aligned}
$$

The lifetime of a rovibronic $\left(\tilde{n}^{\prime} \tilde{\Omega}^{\prime} v^{\prime} J^{\prime} \epsilon^{\prime}\right)$ state is:

$$
\begin{aligned}
& \tau_{\tilde{n}^{\prime} \Omega^{\prime} v^{\prime} J^{\prime} \epsilon^{\prime}} \\
& \quad=\left(\sum_{\tilde{n}^{\prime \prime} \Omega^{\prime \prime} v^{\prime \prime} J^{\prime \prime} \epsilon^{\prime \prime}} A_{\tilde{n}^{\prime} \Omega^{\prime} v^{\prime} J^{\prime} \epsilon^{\prime} ; \boldsymbol{n}^{\prime \prime} \bar{\Omega}^{\prime \prime} v^{\prime \prime} J^{\prime \prime} \epsilon^{\prime \prime}}\right)^{-1} .
\end{aligned}
$$

To carry out the calculations, the $C_{n \tilde{n}}$ coefficients were taken from table 3 of ref. [2]. For the dipole-moment transition elements the ab initio values given in tables 4 and 5 of ref. [1] were used. Moreover, the Franck-Condon factors are assumed to be either 0 (if $v^{\prime} \neq v^{\prime \prime}$ ) or 1 (if $v^{\prime}=v^{\prime \prime}$ ). This is justified because the potential curves in CuF are very nearly parallel (similar $r_{\mathrm{e}}$ and $\omega_{\mathrm{e}}$ ) and because we are merely inter- ested in overall electronic lifetimes. However one should be aware that the model basically attributes zero intensity to $\Delta v \neq 0$ bands. Furthermore it is unable to account for possible though unlikely perturbations that might accidentally occur between interacting high vibrational levels of different electronic states. Anyway the locations of these perturbations are indeterminable in the present status of vibrational data accuracy in CuF. Moreover, on the small range sampled by the vibration, the electronic wavefunction does not change noticeably, thus it was deemed justified to neglect the dependence of the dipole moment operator on the internuclear separation; this would have been an illusionary refinement anyhow as far as even an approximate value of the weak transition dipole moment itself at a given separation is so difficult to obtain in the present case [1]. Under such conditions, the lifetime of a given $n^{\prime} \Omega^{\prime}$ component does not depend on $v^{\prime}$ at all.

The ab initio lifetimes thus obtained are given in table 1 for three different values of $J$ namely $J=5,25$ and 50. In principle, these lifetimes, considered as a function of $J$, should tend towards a rotationless limit equal to the values given in table 7 of ref. [1] in which rotational effects had not been included into the model. However one should notice some deviations due to the use here of new revised wavefunctions [2] following upon the recent discovery of the $A^{3} \Pi_{2}$ component by Brazier, Brown and Steimle [ 8]. This made it possible to add the energy of this state as a new fitting constraint in the determination of the $C_{\tilde{n} n}$ coefficients.

In order to understand the origin of the calculated lifetimes quantitatively, spontaneous emission Einstein coefficients participating in the lifetime values were calculated for each value of the rotational quantum number $J$. An example of such coefficients is given for $J=50$ in table 2 . The set of similar tables for all $J$ values below 100 has been used to calculate rovibronic line intensities within the branches of the $0-0$ bands of the electronic transitions towards the $\mathrm{X}^{1} \Sigma^{+}$ground state. This was carried out as follows. Regarding rovibronic frequencies as constant over a given vibronic transition, the intensity of a line is roughly proportional [3] to the appropriate $A\left(\tilde{n}^{\prime}\right.$, $\left.\tilde{\Omega}^{\prime}, v^{\prime}, J^{\prime}, \epsilon^{\prime} ; \tilde{n}^{\prime \prime}, \tilde{\Omega}^{\prime \prime}, v^{\prime \prime}, J^{\prime \prime}, \epsilon^{\prime \prime}\right)$ Einstein coefficient (such as those given as examples in table 2 for $J^{\prime}=50$ ) and to a Boltzmann exponential factor 
Table 1

Calculated radiative lifetimes (in $\mu$ s) for selected rovibronic $J$ levels of the valence states of $\mathrm{CuF}$

\begin{tabular}{|c|c|c|c|}
\hline & $J=5$ & $J=25$ & $J=50$ \\
\hline${ }^{1} \Delta_{2} \mathrm{e}$ & $2.79 \times 10^{3}$ & $2.99 \times 10^{3}$ & $1.47 \times 10^{3}$ \\
\hline f & $2.79 \times 10^{3}$ & $2.99 \times 10^{3}$ & $1.47 \times 10^{3}$ \\
\hline${ }^{3} \Delta_{1} \mathrm{e}$ & 6.01 & 5.97 & 5.87 \\
\hline f & 6.00 & 5.97 & 5.88 \\
\hline${ }^{3} \Delta_{2} \mathrm{e}$ & $9.86 \times 10^{3}$ & $6.50 \times 10^{3}$ & $3.13 \times 10^{3}$ \\
\hline f & $9.86 \times 10^{3}$ & $6.50 \times 10^{3}$ & $3.14 \times 10^{3}$ \\
\hline${ }^{3} \Delta_{3} \mathrm{e}$ & $14.4 \times 10^{3}$ & $12.1 \times 10^{3}$ & $8.0 \times 10^{3}$ \\
\hline f & $14.4 \times 10^{3}$ & $12.1 \times 10^{3}$ & $8.0 \times 10^{3}$ \\
\hline $\mathrm{C}^{1} \Pi \mathrm{e}$ & 0.400 & 0.401 & 0.403 \\
\hline f & 0.399 & 0.400 & 0.401 \\
\hline $\mathrm{B}^{1} \Sigma^{+} \mathrm{e}$ & 1.23 & 1.22 & 1.21 \\
\hline$A^{3} I_{0} e$ & 5.56 & 5.85 & 6.39 \\
\hline f & $733 \times 10^{3}$ & $90.8 \times 10^{3}$ & $24.7 \times 10^{3}$ \\
\hline $\mathrm{A}^{3} \Pi_{1} \mathrm{e}$ & 13.9 & 12.4 & 10.5 \\
\hline f & 14.6 & 14.6 & 14.6 \\
\hline $\mathrm{A}^{3} \mathrm{II}_{2} \mathrm{e}$ & $417 \times 10^{3}$ & $17.5 \times 10^{3}$ & $4.39 \times 10^{3}$ \\
\hline f & $418 \times 10^{3}$ & $17.7 \times 10^{3}$ & $4.53 \times 10^{3}$ \\
\hline$a^{3} \Sigma_{0}^{+} \mathbf{f}$ & 4893 & 505 & 330 \\
\hline$a^{3} \Sigma_{1}^{+} \mathbf{e}$ & 99 & 99 & 98 \\
\hline f & 101 & 122 & 140 \\
\hline
\end{tabular}

$\exp [-h c F(J) / k T]$ where $F(J)$ is the rotational term value ( $J^{\prime}$ for emissions $J^{\prime \prime}$ for absorption) and $T$ is the absolute temperature taken equal to $300 \mathrm{~K}$ as assumed by Ahmed et al. [9] for their hollow-cathode emission spectra. Inspection of tables 1 and 2 shows that all transition probabilities between states both of which belong to the $\mathrm{Cu}^{+}\left(3 \mathrm{~d}^{9} 4 \mathrm{~s}\right)$ manifold are exceedingly small in comparison with most transition probabilities between these states and the ground state. Therefore, as for their lifetimes, it is convenient to consider three classes of states: the short-lived states $\left(B^{1} \Sigma^{+}\right.$and $\left.C^{1} \Pi\right)$ with lifetimes shorter than or around $1 \mu \mathrm{s}$, the mean-lived states $(\Omega=0 \mathrm{e}$ and $\Omega=1$ components) with lifetimes in the 5-500 $\mu$ s range, and the long-lived states ( $\Omega=0$ f and $\Omega \geqslant 2$ ) with lifetimes above $1 \mathrm{~ms}$. These three classes will be successively discussed in the next sections.

\section{Short-lived states}

The $\mathrm{B}^{1} \Sigma^{+}$and $\mathrm{C}^{1} \Pi$ states are the only ones to be able to radiate towards the $\mathrm{X}^{1} \Sigma^{+}$ground state within the purely electronic approximation. Although they are somewhat longer than usually found when electronic transitions are allowed, the lifetimes of these essentially singlet states are nevertheless at least one order of magnitude shorter than those of the other states of the $\mathrm{Cu}^{+}\left(3 d^{9} 4 s\right)$ structure. The part of Coriolis interactions in the $\mathrm{B}^{1} \Sigma^{+}$and $\mathrm{C}^{1} \Pi$ wavefunctions brings no significant relative contribution to the lifetime values which are therefore nearly constant whatever $J$. Accordingly the discussion about the radiative decay mechanisms of the $B$ and $C$ states as given in ref. [ 1 ] still remains valid: the observed values of the lifetimes are due to a subtle conjugation of orbital-polarization, dynamic-rearrangement and configuration-interaction effects. The look of a typical spectroscopic band ( $0-0$ band) for of the C-X and $B-X$ systems is given in figs. 1 and 2 . The intensity distributions do not exhibit marked anomalies except that the $R$ branch appears to be a little less intense than the $P$ branch in the $B-X$ transition, due to interference effects involving the ${ }^{3} \Pi$ contamination in $B^{1} \Sigma^{+}$. It is interesting to notice that these systems are the most intense observed ones in CuF. Nevertheless their electronic oscillator strengths are only 0.01 for $\mathrm{C}-\mathrm{X}$ and $\mathbf{0 . 0 0 4}$ for B-X. This means that other intense electronic transitions should exist, for instance $4 s \rightarrow 4 p$ ones, but these transitions have not yet been observed.

\section{Mean-lived states}

The $D^{3} \Delta_{1}, A^{3} \Pi_{1}, a^{3} \Sigma_{1}^{+}$and $A^{3} \Pi_{0 e}$ states constitute the second class. Their transition probabilities towards the ground state result from spin-orbit interactions with $C^{1} \Pi$ for the first three states or with $B^{1} \Sigma^{+}$for the ${ }^{3} \Pi_{0 e}$ state. It should be noticed that, although it is small, the ${ }^{3} \Pi_{0 e}$ character of the $X^{1} \Sigma^{+}$ ground state also contributes for about $10 \%$ in the $A^{3} \Pi_{0 e}-X^{1} \Sigma^{+}$transition probability as a result of the large value of the diagonal ${ }^{3} \Pi$ dipole moment [ 1 ]. 


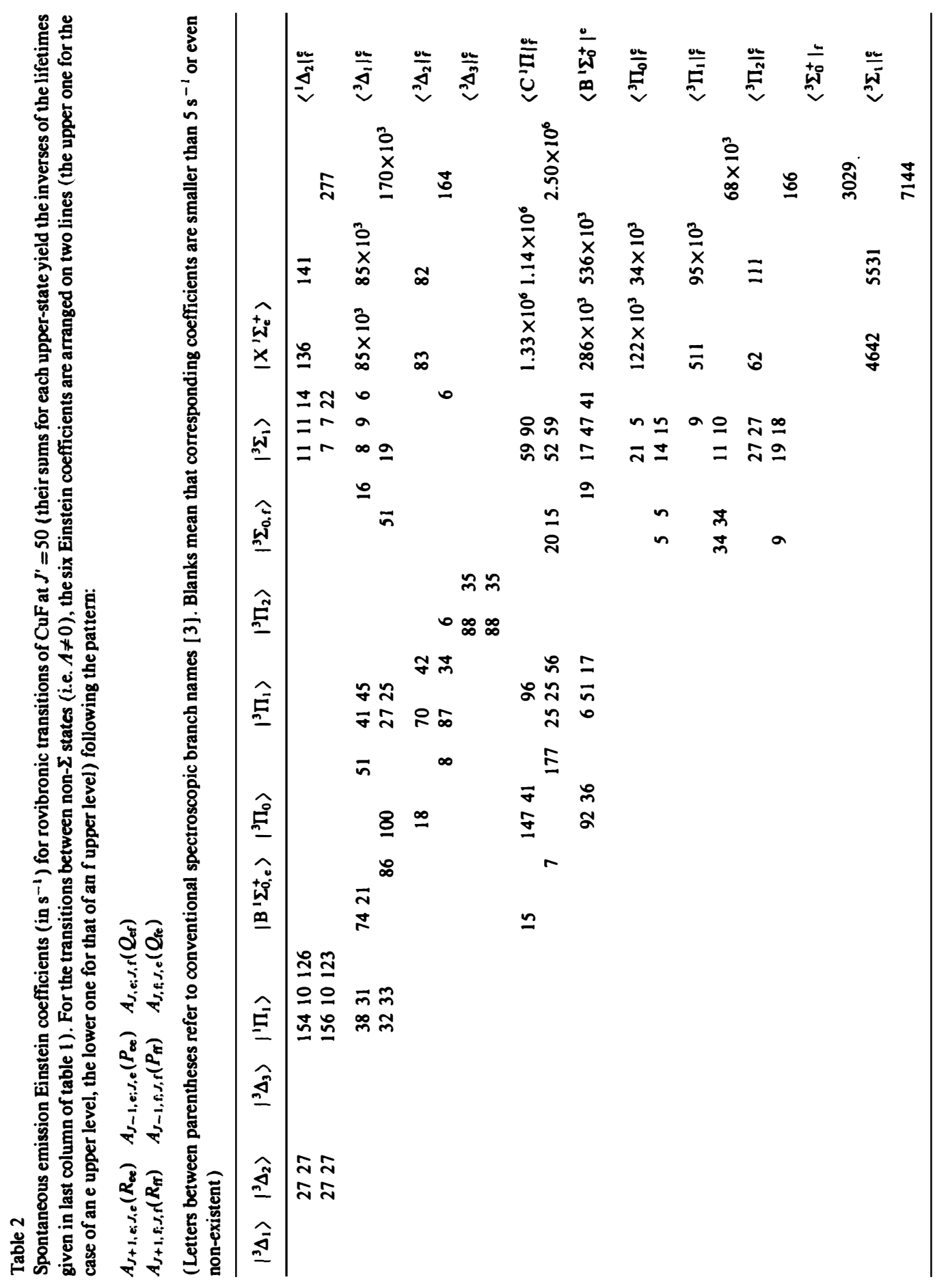




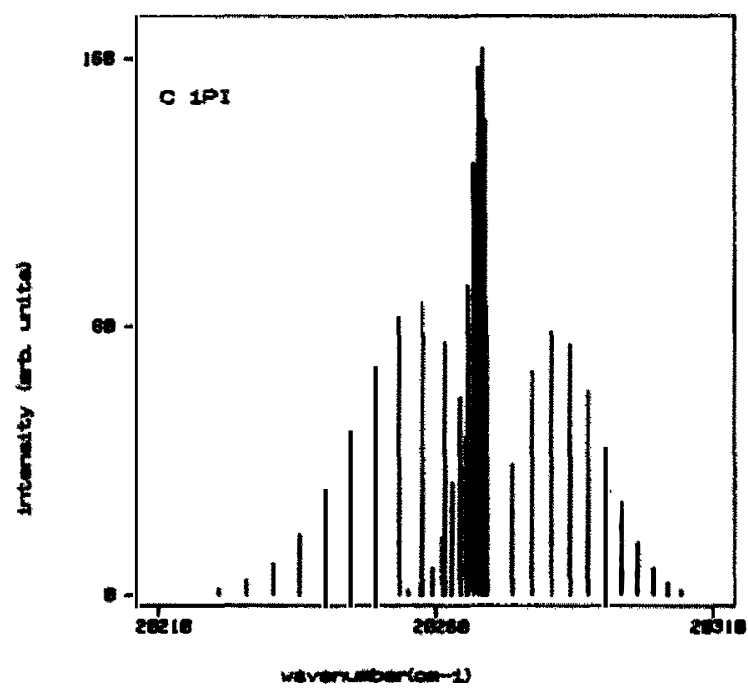

Fig. 1. Calculated intensity distribution in branches of the $0-0$ band of $C^{\prime} \Pi-X^{\prime} \Sigma^{*} . T=300 \mathrm{~K}$; only every fifth rotational line is depicted. Intensity units are arbitrary but they are the same for all figures. Wavenumber scales are given for information only since the model does not include second-order corrections such as centrifugal distortion.

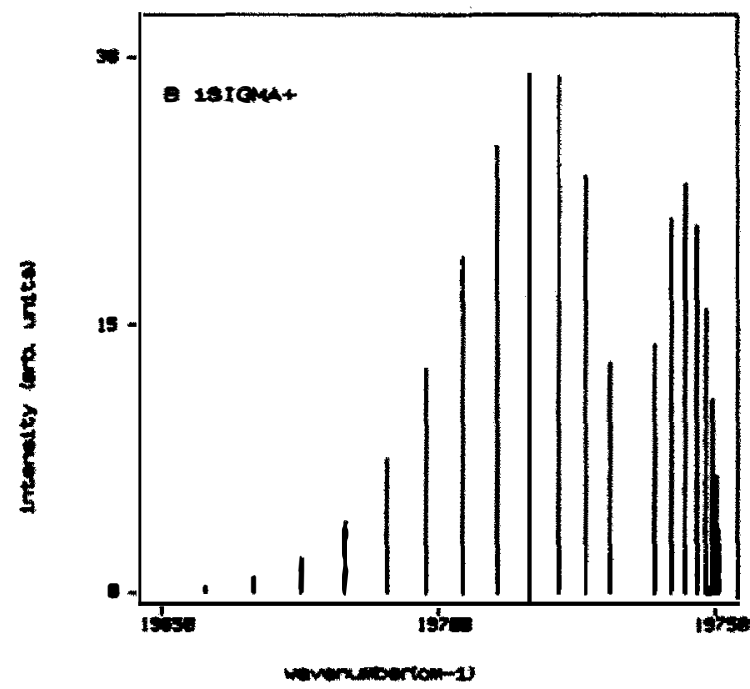

Fig. 2. Calculated intensity distribution in branches of the 0-0 band of $B^{1} \Sigma^{+}-X^{\prime} \Sigma^{+}$(see caption of fig. 1 ).

Note also that because of their accidental quasi-degeneracy the $A^{3} \Pi_{0 e}$ and $A{ }^{3} \Pi_{1 e}$ states mix through rotational spin-uncoupling so that their lifetimes, no- ticeably different at low $J$, get closer to each other as $J$ increases and should tend to a common value at much higher $J$. On the other hand, the ${ }^{3} \Pi_{1 f}$ component, which is much farther (at least ten times) in energy from the ${ }^{3} \Pi_{\text {or }}$ one than ${ }^{3} \Pi_{1 e}$ is from ${ }^{3} \Pi_{0 e}$, keeps a lifetime that does not depend on $J$. It would be interesting to carry out rotationally resolved lifetime measurements so as to try and observe these effects. Similar comments apply to the case of the $a^{3} \Sigma^{+}$state: since there is no e component for a ${ }^{3} \Sigma_{0}^{+}$state, the $a^{3} \Sigma_{i c}^{+}$component has a lifetime that does not depend on $J$ whereas the progressively enhanced rotational mixing between the $\mathrm{A}^{3} \Sigma_{\text {If }}^{+}$and $\mathrm{A}^{3} \Sigma_{\text {of }}^{+}$components leads to $J$-dependent lifetimes for these f components and makes spectroscopic observation of the ${ }^{3} \Sigma_{0}^{+}$ component possible [10]. Lastly note that the present calculations gratifyingly account for the intensity anomalies (with respect to the standard $2: 1: 1$ ratio for $\mathbf{Q}: \mathbf{P}: \mathbf{R}$ branch intensities) found by Ahmed et al. [9] in the branches of the A-X transition. For example, as illustrated in figs. 3 and 4 the calculations satisfactorily render the fact that the R-branch intensity is higher than the P-branch one (by a factor of two at $J=35$ ) in the $A^{3} \Pi_{0 e}-X^{1} \Sigma^{+}$subband while on the contrary the P-branch intensity is much higher than the R-branch one (by a factor of five at $J=20$ ) in the $A^{3} \Pi_{1}-X^{1} \Sigma^{+}$subband. Moreover another ex-

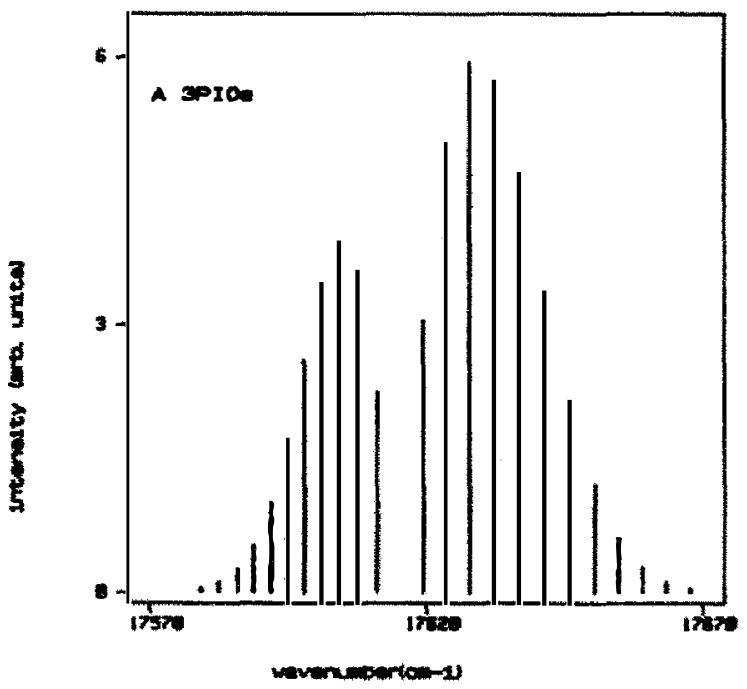

Fig. 3. Calculated intensity distribution in branches of the $0-0$ band of $A^{3} \Pi_{0 e}-X^{1} \Sigma^{+}$( see caption of fig. 1). 


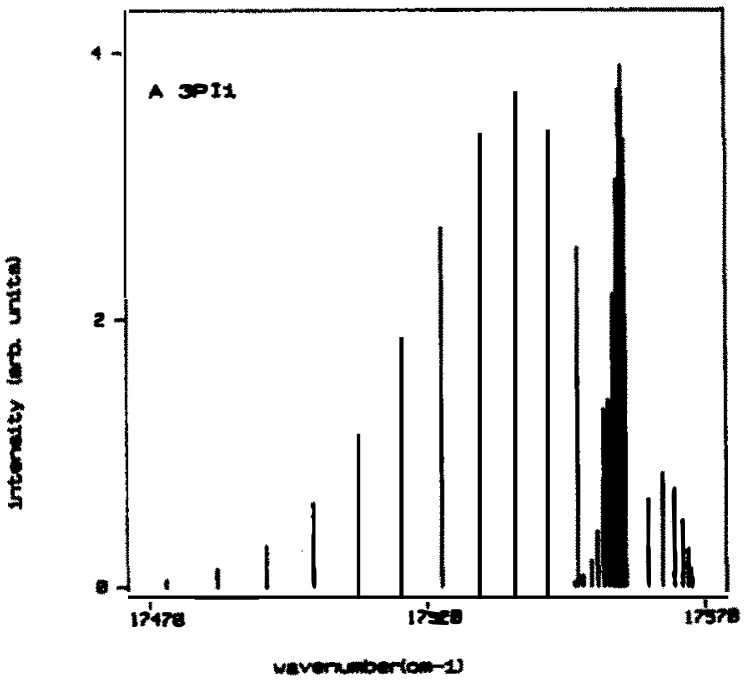

Fig. 4. Calculated intensity distribution in branches of the $0-0$ band of $A^{3} \Pi_{1}-X^{1} \Sigma^{+}$( see caption of fig. 1).

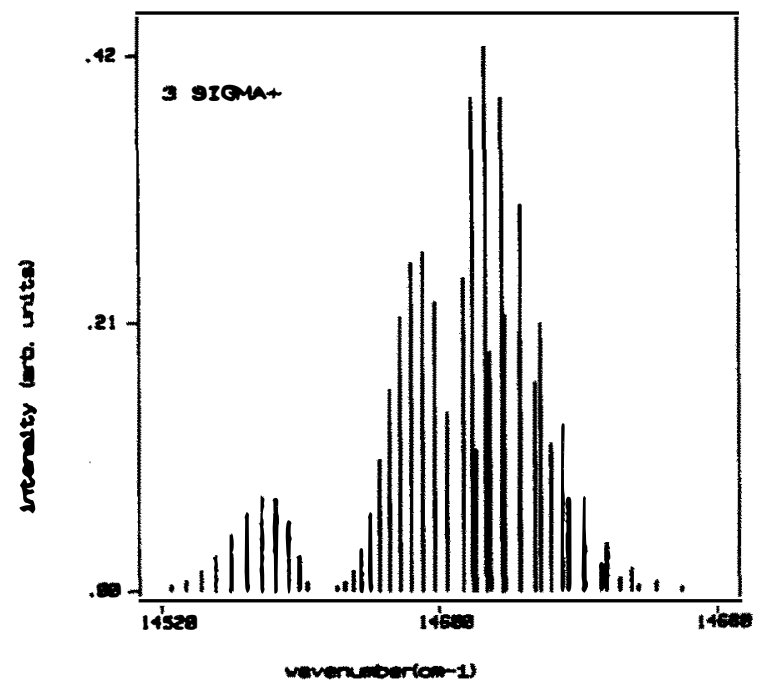

Fig. 5. Calculated intensity distribution in branches of the 0-0 band of a ${ }^{3} \Sigma^{+}-X^{1} \Sigma^{+}$(see caption of fig. 1).

perimentally observed feature namely the fact that intensity maximum in the R-branch occurs at lower $J$ value (about ten units less) than in the $P$ and $Q$ branches of the $A^{3} \Pi_{1}-X^{1} \Sigma^{+}$subband, is correctly reproduced by the calculations. Similar calculated intensity patterns for the a ${ }^{3} \Sigma^{+}-X^{1} \Sigma^{+}$and $D^{3} \Delta_{1}-X^{1} \Sigma^{+}$ transitions are given in figs. 5 and 6 respectively. As

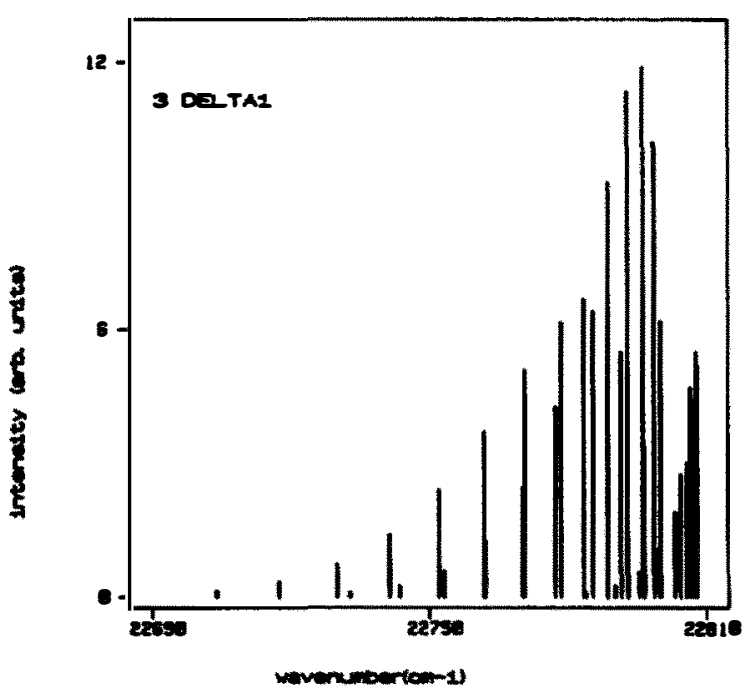

Fig. 6. Calculated intensity distribution in branches of the $0-0$ band of $D^{3} \Delta_{1}-X^{1} \Sigma^{+}$(see caption of fig. 1).

far as they can be compared with the spectrum photographic reproduction given by Ahmed et al. [9], the relative branch intensities in fig. 5 are in good agreement with experiment.

\section{Long-lived states}

The a ${ }^{3} \Sigma_{0}^{+}, \mathrm{A}^{3} \mathrm{IL}_{\text {of }}$ components and all the $\Omega=2$ and $\Omega=3$ components, i.e. $\mathrm{A}^{3} \Pi_{2}, \mathrm{D}^{3} \Delta_{2}, \mathrm{D}^{3} \Delta_{3}$ and ${ }^{1} \Delta_{2}$ constitute the third class. In the non-rotating approximation, these states cannot radiate towards the $X^{\prime} \Sigma^{+}$ ground state but only towards other excited states with transition probabilities smaller by several orders of magnitude [1] than those transition probabilities considered above for the $\Omega=0 \mathrm{e}$ and $\Omega=1$ components. This constitutes a first radiative decay mechanism.

Taking into account rotational motion brings some ${ }^{1} \Pi_{1}$ character into the wavefunctions of these metastable states and therefore leads to a second radiative decay mechanism that takes place towards the ground state and accordingly makes the corresponding lifetime a decreasing function of $J$.

For so weak electric-dipolar transition probabilities it was deemed necessary to ascertain that a third mechanism, namely electric quadrupolar radiation, 
could still be neglected. This is all the more important as the observed molecular transitions in $\mathrm{CuF}$ have been shown [10] to be connected with electricdipole-forbidden but electric-quadrupole-allowed 3d$4 \mathrm{~s}$ transitions in the $\mathrm{Cu}^{+}$atomic limit. The as yet unobserved ${ }^{\prime} \Delta-X^{1} \Sigma^{+}$bands which are due to a spinallowed dipolar-electronically forbidden transition are particularly concerned. Using previously determined orbital wavefunctions [1] an ab initio calculation of the associated quadrupole-electronic-transition moment was carried out. The obtained value was small, about 0.5 (in $a_{0}^{2} e$ units), from which it can be deduced [11] a quadrupolar spontaneous emission probability of only $0.033 \mathrm{~s}^{-1}$. This means that quadrupolar radiation can be neglected compared to the two first mechanisms quoted above, namely dipole-allowed electronic transitions between close-lying excited states competing with Coriolis-induced transitions towards the ground state.

Which of these two mechanisms dominates depends on the state under consideration. For instance, the radiative decay of the $A^{3} \Pi_{0 f}$ and $D^{3} \Delta_{3}$ states is essentially due to the first mechanism (electronically allowed transitions): the $A^{3} \Pi_{0 f}$ state decays toward $a^{3} \Sigma_{1}^{+}$and the $D^{3} \Delta_{3}$ state towards $A^{3} \Pi_{2}$. It is worthwhile noting that the lifetimes of the $A^{3} \Pi_{\text {of }}$ and $D^{3} \Delta_{3}$ states are nevertheless strongly $J$-dependent. This unexpected effect results from Coriolis contamination of the wavefunctions. Although the - J-dependent $-C_{n^{\prime} n}$ coefficients due to this contamination are weak, they introduce terms involving large diagonal dipole-moment elements in the calculations of the line-strengths (see above the expression for $S$ ), so large that, in net quantitative magnitude, these terms outmatch the supposedly first-order $J$-independent contributions of the very weak ${ }^{3} \Pi-{ }^{3} \Sigma^{+}$and ${ }^{3} \Delta-{ }^{3} \Pi$ electronic transition moments. It is also because of the predominant part of these diagonal dipole-moment elements that the as yet unobserved $\mathrm{A}^{3} \Pi_{0 \mathrm{O}^{-}} \mathrm{a}^{3} \Sigma_{1}^{+}$and $\mathrm{D}^{3} \Delta_{3}-\mathrm{A}^{3} \Pi_{2}$ transitions should be expected to exhibit completely anomalous branch intensity patterns (see table 2 ) with a very strong asymmetry between $P$ and $R$ branches. In any case, the transition probabilities between both $A^{3} \Pi_{\text {of }}$ and $\mathrm{D}^{3} \Delta_{3}$ upper states and the $\mathrm{X}^{1} \Sigma^{+}$ground state are calculated to be exceedingly small and, contrary to Brazier's expectations for $A^{3} \Pi_{\text {of }}[8]$, it is undoubtedly impossible to observe the corresponding transi- tions through even sophisticated usual spectroscopic methods. On the opposite, as already mentioned in section 4 , radiative relaxation of the $a^{3} \Sigma_{0}^{+}$state is only due to spin-uncoupling mixing with the closelying a ${ }^{3} \Sigma_{1}^{+}$state from which it borrows the ${ }^{1} \Pi_{1}$ character necessary to radiate towards the ground state. Lastly, radiative relaxation of the three $\Omega=2 \mathrm{com}$ ponents takes place through both mechanisms: not only do they decay towards lower-lying states of the same $\mathrm{Cu}^{+}\left(3 \mathrm{~d}^{9} 4 \mathrm{~s}\right)$ structure $\left({ }^{1} \Delta_{2}\right.$ towards $\mathrm{D}^{3} \Delta_{2}$ and $\mathrm{C}^{1} \Pi_{1} ; \mathrm{D}^{3} \Delta_{2}$ towards $\mathrm{A}^{3} \Pi_{1} ; \mathrm{A}^{3} \Pi_{2}$ towards a ${ }^{3} \Sigma_{1}^{+}$) but as soon as $J$ reaches sufficiently high values, these three $\Omega=2$ components can also decay towards the $\mathrm{X}^{1} \Sigma^{+}$ground state, owing to their weak ${ }^{1} \Pi_{1}$ character borrowed through rotational uncoupling. Thus, at $J=50$, this last mechanism participates for $40 \%$ in the ${ }^{1} \Delta_{2}$ lifetime, for $50 \%$ in the $\mathrm{D}^{3} \Delta_{2}$ one and for $75 \%$ in the $A^{3} \Pi_{2}$ one.

Indeed this ${ }^{3} \Pi_{2}$ state has been observed via laserexcitation spectroscopy [8], a now usual experimental method in which $\mathrm{X}^{1} \Sigma^{+} \rightarrow \mathrm{A}^{3} \Pi_{2}$ absorption is detected on looking at the overall light reemitted after more or less complex radiative and collision processes. Fig. 7 represents the calculated intensity pattern of the $0-0$ band of this $A^{3} \Pi_{2}-X^{1} \Sigma^{+}$system. The ratio between the intensities of the $Q$ and $R$ branches is satisfactorily found to be the same as in Brazier et

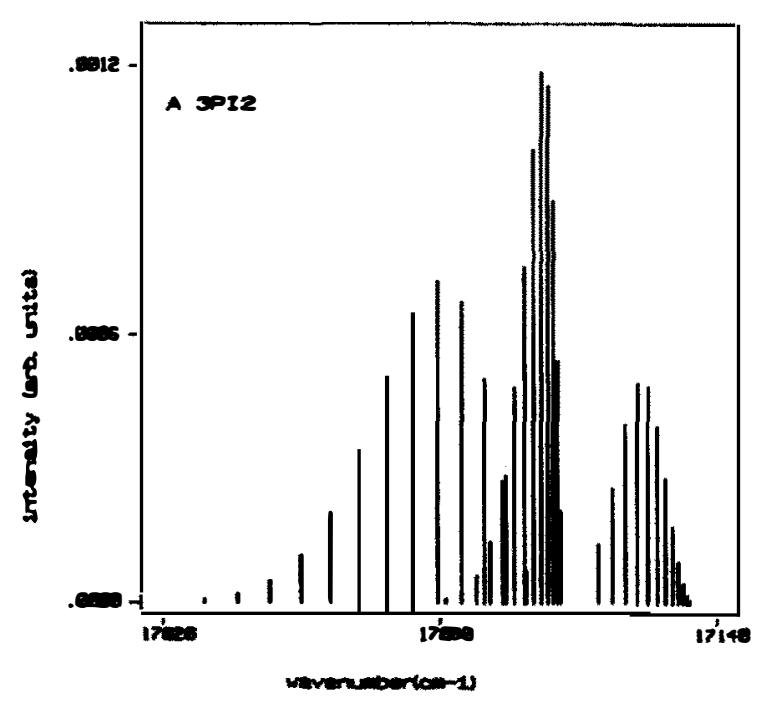

Fig. 7. Calculated intensity distribution in branches of the 0-0 band of $A^{3} \Pi_{2}-X^{1} \Sigma^{+}$( see caption of fig. 1 ). 
al.'s spectroscopic observations [8]. But there appears a drastic discrepancy as far as the $P$ branch is considered. This raises an interesting point that deserves special discussion. Brazier et al. [8] report observation of some very faint $P$ lines they estimate to be about five times less intense than corresponding $R$ lines. On the contrary, present calculations predict Pbranch intensity to be nearly equal to and even slightly more intense than R-branch intensity. Besides this could only but be expected inasmuch as the $\mathrm{A}^{3} \mathrm{II}_{2-}$ $\mathrm{X}^{1} \Sigma^{+}$transition mainly gets its intensity from the $A^{3} \Pi_{1}-X^{1} \Sigma^{+}$one through Coriolis interaction so that it should reflect the P-over- $R$ intensity preponderance both observed and calculated for this band system (section 4, fig. 4). Admittedly this preponderance is much attenuated in the $A^{3} \Pi_{2}-X^{1} \Sigma^{+}$ transition where interference effects are less marked than in the $A^{3} \Pi_{1}-X^{1} \Sigma^{+}$one. But at any rate, from detailed analysis of the contributions to branch intensities, $\mathbf{P}-\mathbf{R}$ intensity inversion from one system to the other definitely cannot occur within the present model. On view of this one could indeed wonder whether a limit of this model has not been reached in this particularly delicate case. However extension of the model via admixture of the weak [1] configuration interaction coming from structures other than $\left(3 d^{9} 4 s\right)$, for instance $\left(3 d^{9} 4 p\right)$, does not bring any significant change: as they stand, the results are remarkably stable with respect to manageable improvement of the model. Therefore it is necessary to question about the possibility of an accidental artefact that would arise from the use of so tricky a method as laser excitation spectroscopy and that would accordingly invalidate comparison between calculated and observed intensities. Indeed it should be noticed that it is odd to say the least that the Pbranch intensity is so low whereas that of the $R$ branch is normal as compared to the Q-branch one. This artefact could not be asymmetric quenching depopulation of the excited levels since both $P$ and $R$ lines, which necessarily originate from the same e rovibronic levels, would be affected in exactly the same way. But it could be a selective radiative depopulation effect: one could imagine for instance a two-step $\mathrm{X}^{1} \Sigma^{+}-\mathrm{A}^{3} \Pi_{2} \rightarrow$ ? process whose second step would be resonant when the laser pump is set at P-line wavelengths but off-resonant when it is at R-line wavelengths. For P-line pumping the probe fluorescence would then be mainly ultraviolet radiation (possibly out of the detector range) or even missing if higher states are dissociative or predissociated while it would be (well detected) visible radiation for $R$-line pumping. The question of the $A^{3} \Pi_{2}-X^{1} \Sigma^{+}$intensity pattern remains open and new experiments would be welcome.

From our calculations, the $\mathrm{X}^{1} \Sigma^{+}-{ }^{1} \Delta_{2}$ and $\mathrm{X}^{1} \Sigma^{+}-$ $D^{3} \Delta_{2}$ transition moments are predicted to be of similar (weak) magnitude as that of the $X^{1} \Sigma^{+}-A^{3} \Pi_{2}$ one (calculated values at $J=50$ : respectively 2,3 and $4 \times 10^{-3} \mathrm{au}$ ). Figs. 8 and 9 show the calculated intensity patterns for these two transitions that again appear to have standard appearance. Moreover due to their higher frequency they are expected to be respectively four times and twice as intense as the $A^{3} \Pi_{2-}$ $\mathrm{X}^{1} \Sigma^{+}$transition. Therefore the present calculations should give an impetus to spectroscopists in order to try and detect the ${ }^{1} \Delta_{2}$ and $D^{3} \Delta_{2}$ states by the same method as the one used to discover the $A^{3} \Pi_{2}$ state [8], i.e. laser excitation spectroscopy from the ground state even though the predicted spectral regions [10] are experimentally less easy to reach.

\section{Conclusion}

This calculation of rotationally resolved lifetimes

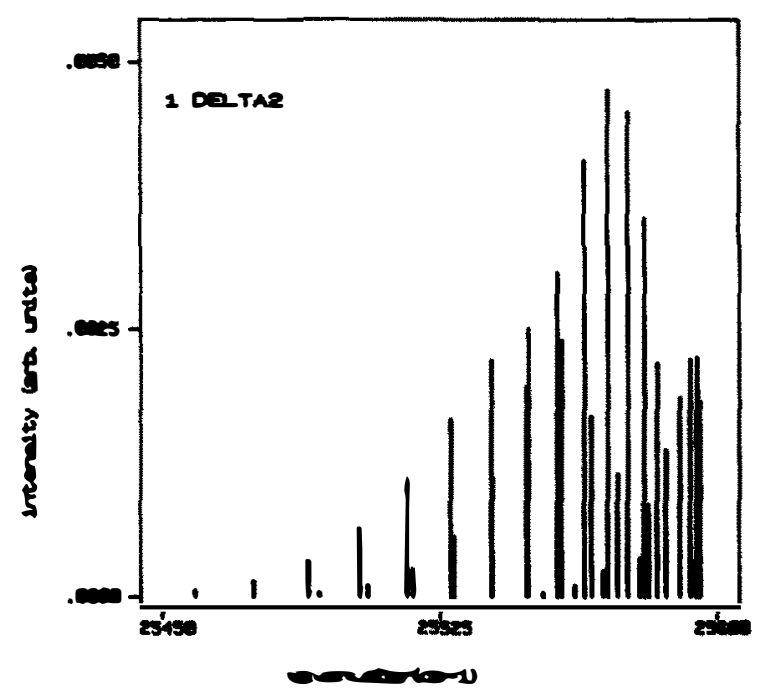

Fig. 8. Calculated intensity distribution in branches of the $0-0$ of ${ }^{\prime} \Delta_{2}-X^{\prime} \Sigma^{+}$( see caption of fig. 1 ). 


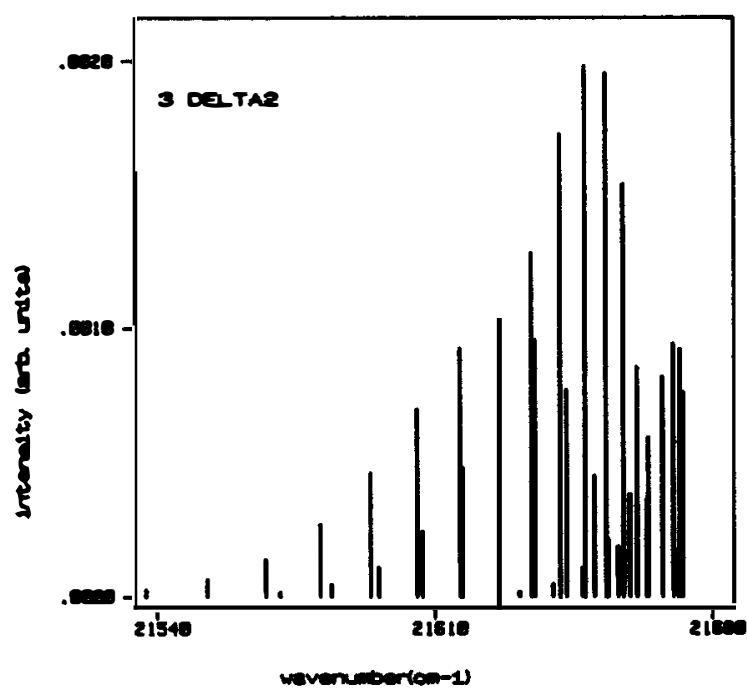

Fig. 9. Calculated intensity distribution in branches of the $0-0$ band of ${ }^{3} \Delta^{+}$( see caption of fig. 1 ).

in CuF provides a quantitative understanding of the relative importance of the various mechanisms that govern radiative relaxation of the rovibronic levels of the $\mathrm{Cu}^{+}\left(3 d^{9} 4 s\right) F^{-}\left(2 p^{6}\right)$ excited electronic states. Up to now all the observed spectroscopic transitions of CuF involve components that belong to this ionic structure although these transitions would be electronically forbidden in the copper atom limit due to their $\Delta l=2$ nature. Molecular symmetry lifts this orbital restriction and this is the origin of the moderately short $(<1 \mu \mathrm{s})$ lifetime of the $\mathrm{B}^{1} \Sigma^{+}$and $\mathrm{C}^{1} \Pi$ states. Surprisingly enough, this appears more because of configuration interaction and dynamic orbital rearrangements than because of structural orbital hybridization [1]. In a second step, spin-orbit interaction mixes triplet components with singlet ones of same $\Omega$ value and this explains the occurrence of most of the other observed emissions. Their upper states exhibit moderately long ( $\approx 6$ to $100 \mu \mathrm{s}$ ) and rotationally independent lifetimes. If one excludes the $\mathrm{a}^{3} \Sigma_{0}^{+}$component that is observed because of its $\mathrm{mix}-$ ing with the close-lying a ${ }^{3} \Sigma_{1}^{+}$one, the only observed state outside these two classes is the ${ }^{3} \Pi_{2}$ component. It appears in a ${ }^{3} \Pi_{2}-X^{1} \Sigma^{+}$transition made allowed owing to Coriolis coupling that gives the rotational levels of the ${ }^{3} \mathrm{II}_{2}$ upper state a long ( $5 \mathrm{~ms}$ at $J=50$ ) rotationally dependent radiative lifetime. This ${ }^{3} \Pi_{2}$ state is the only observed member of the class of the so-called long-lived states. Excluding the at least two orders of magnitude weaker quadrupolar radiation, the magnitudes of their rotationally dependent lifetimes should be ascribed to two competing mechanisms linked to Coriolis contamination that allows radiation towards the ground state or enhances in a subtle way the decay towards other excited states. These effects are weak but nevertheless significant. Thus spectroscopic observation of the ${ }^{1} \Delta_{2}$ and $D^{3} \Delta_{2}$ states via laser excitation from the ground state does not seem impossible at all since the corresponding transition probabilities are calculated to be of the same magnitude as that of the already observed $\mathrm{A}^{3} \mathrm{II}_{2}-\mathrm{X}^{1} \Sigma^{+}$transition. On the other hand, forecasts for discovery of the $\mathrm{D}^{3} \Delta_{3}$ and $\mathrm{A}^{3} \mathrm{II}_{0}$ of states by this method are definitely pessimistic and those components are likely to escape observation for a long time yet. Table 3 summarizes the calculated relative intensities of the most intense lines of each system. This table plainly confirms in a quantitative way the qualitative estimates about relative observed intensities given by Ahmed et al. [9] and it predicts intensities of the as yet unobserved transitions.

To conclude, it should be emphasized that, at the scale of the lifetimes involved here, non-radiative transfers are likely to play a fundamental part in actual processes involving the excited states of gas phase $\mathrm{CuF}$ even at low pressure. They are expected to drastically bring down effective lifetimes of the long-lived

Table 3

Summary of calculated intensities of the electronic transitions of $\mathrm{CuF}$

\begin{tabular}{|c|c|c|}
\hline Band system & $\begin{array}{l}\lambda(0-0 \text { band }) \\
(\mathrm{nm})\end{array}$ & $\begin{array}{l}\text { Calculated } \\
\text { intensity } \\
\text { (arb. units) }\end{array}$ \\
\hline${ }^{1} \Delta_{2}-X^{\prime} \Sigma^{+}$ & $(395)$ & $4 \times 10^{-3}$ \\
\hline$D^{3} \Delta_{1}-X^{1} \Sigma^{+}$ & 438.6 & 12 \\
\hline$D^{3} \Delta_{2}-X^{1} \Sigma^{+}$ & $(460)$ & $2 \times 10^{-3}$ \\
\hline$D^{3} \Delta_{3}-X^{1} \Sigma^{+}$ & (473) & $7 \times 10^{-7}$ \\
\hline$C^{\prime} \Pi_{1}-X^{\prime} \Sigma^{+}$ & 493.4 & 160 \\
\hline $\mathrm{B}^{1} \Sigma_{0}^{+}-\mathrm{X}^{1} \Sigma^{+}$ & 506.7 & 30 \\
\hline$A^{3} \Pi_{0 r} X^{1} \Sigma^{+}$ & $(548)$ & $3 \times 10^{-5}$ \\
\hline$A^{3} \Pi_{1}-X^{1} \Sigma^{+}$ & 567.8 & 3 \\
\hline$A^{3} \Pi_{0 e}-X^{1} \Sigma^{+}$ & 569.6 & 5 \\
\hline$A^{3} \Pi_{2}-X^{1} \Sigma^{+}$ & 584.6 & $1 \times 10^{-3}$ \\
\hline$a^{3} \Sigma_{1}^{+}-X^{1} \Sigma^{+}$ & 684.6 & 0.4 \\
\hline$a^{3} \Sigma_{0}^{+}-X^{1} \Sigma^{+}$ & 686.7 & 0.1 \\
\hline
\end{tabular}


states and they may appreciably depopulate these states long before they radiate. Calculation of nonradiative state-to-state transfer rates would be quite useful for a complete assessment of the dynamical processes governing the behaviour of $\mathrm{CuF}$ excited states. However, for practical reasons this is out of the question for the time being.

\section{References}

[1] J.M. Delaval and J. Schamps, Chem. Phys. 100 (1985) 21.

[2] J.M. Delaval, J. Schamps and C. Dufour, J. Mol. Spectry. 137 (1989) 268.

[3] G. Herzberg, Spectra of Diatomic Molecules (Van Nostrand, Princeton, 1950).
[4] E.E. Whiting and R.W. Nicholls, Astrophys. J. Suppl. 27 (1974) 1 .

[5] E.E. Whiting, A. Schadee, J.B. Tatum, J.T. Hougen and R.W. Nicholls, J. Mol. Spectry. 80 (1980) 249.

[6] M. Larsson, Astron. Astrophys. 128 (1983) 291.

[7] H. Lefebvre-Brion and R.W. Field, Perturbations in the Spectra of Diatomic Molecules (Academic Press, New York, 1986).

[8] C. Brazier, J.M. Brown and T.C. Steimle, J. Mol. Spectry. 97 (1983) 449.

[9] F. Ahmed, R.F. Barrow, A.H. Chojnicki, C. Dufour and J. Schamps, J. Phys. B 15 (1983) 3801.

[10] C. Dufour, J. Schamps and R.F. Barrow, J. Phys. B 15 (1982) 3819.

[11] B.W. Shore and D.H. Menzel, Principles of Atomic Spectra (Wiley, New York, 1968 ). 